\title{
The Proper Forcing Axiom and the Singular Cardinal Hypothesis
}

\author{
Matteo Viale *
}

\begin{abstract}
We show that the Proper Forcing Axiom implies the Singular Cardinal Hypothesis. The proof uses the reflection principle MRP introduced by Moore in [10].
\end{abstract}

\section{Introduction}

In one of the first applications of the forcing techniques, Easton [3] showed that the exponential function $\kappa \mapsto 2^{\kappa}$ on regular cardinals can be arbitrary modulo some mild restrictions. The situation for singular cardinals is much more subtle. For instance, Silver [12] showed that the Singular Cardinal Hypothesis SCH cannot first fail at a singular cardinal of uncountable cofinality. Recall that $\mathrm{SCH}$ states that $2^{\kappa}=$ $\kappa^{+}$, for all singular strong limit cardinals $\kappa$. However, it is known that $\mathrm{SCH}$ can first fail even at $\aleph_{\omega}$.

The role of large cardinals in this context is twofold. On one hand they are necessary for the construction of models of the negation of $\mathrm{SCH}$ since any such model has an inner model with measurable cardinals (see [5] for a survey of Prikry type forcings and applications to $\mathrm{SCH}$ ). On the other hand it is a theorem of Solovay [13] that $\mathrm{SCH}$ holds above a strongly compact cardinal. Forcing axioms imply reflection principles similar to the one used in Solovay's proof, thus it

\footnotetext{
0 2000 Mathematical Subject Classification: 03E05, 03E10, 03E65, 03E75. Key words: MRP, PFA, SCH.

* Dipartimento di Matematica, Universitá di Torino and Equipe de Logique Mathématique, Université Paris 7, email: viale@dm.unito.it
} 
was reasonable to expect that they would also settle $\mathrm{SCH}$. Indeed, in [4], Foreman, Magidor and Shelah showed that the strongest forcing axiom, Martin's Maximum MM, implies SCH. This was later improved by Veličković [14] who also showed that $\mathrm{SCH}$ follows from $\mathrm{PFA}^{+}$. In fact, what is shown in [14] is that if $\theta>\aleph_{1}$ is regular and stationary subsets of $[\theta]^{\omega}$ reflect to an internally closed and unbounded set, then $\theta^{\omega}=\theta$. This, combined with Silver's theorem, implies SCH. At this point, it was left open whether SCH is a consequence of PFA.

Very little progress was made on this problem for over fifteen years. Then, in 2003, Moore [10] introduced a new reflection principle, the Mapping Reflection Principle MRP and deduced it from PFA. He showed that MRP implies the continuum is equal to $\aleph_{2}$ and the failure of $\square(\kappa)$, for all $\kappa>\aleph_{1}$. MRP has many features in common with the reflection principles which follow from $M M$, so it should be expected that MRP could affect the behaviour of the exponential function also on higher cardinals. In fact, Moore showed in [11] that if MRP holds and $\kappa>\omega_{1}$ is a regular cardinal with a nonreflecting stationary set consisting of points of countable cofinality, then $\kappa^{\omega_{1}}=\kappa$. This, combined with the above result of Veličković, strongly suggests that PFA implies SCH. In this paper we confirm this conjecture.

The paper is organized as follows. In section $\S 1$ we prove that if MRP holds then $\kappa^{\omega}=\kappa$ for all regular cardinals $\kappa>\omega_{1}$. This, together with Silver's theorem, implies that SCH follows from PFA. In the final section we show that the result can be refined and applied to study another interesting problem in the area of forcing axioms, i.e. to investigate what kind of forcing notions can preserve this type of axioms.

Our notation is standard and follows [7] and [10]. For a regular cardinal $\theta$, we use $H(\theta)$ to denote the structure $(H(\theta), \in,<)$ whose domain is the collection of sets whose transitive closure is of size less than $\theta$ and where $<$ is a predicate for a fixed well ordering of $H(\theta)$. If $X$ is an uncountable set, $C \subseteq[X]^{\omega}$ is closed and unbounded (club) if there is $f:[X]^{<\omega} \rightarrow X$ such that $C$ is the set of all $Y \in[X]^{\omega}$ such that $f[Y]^{<\omega} \subseteq Y . S \subseteq[X]^{\omega}$ is stationary if it intersects all club subsets of $[X]^{\omega}$. The $f$-closure of $X$ is the smallest $Y$ containing $X$ such that $f[Y]^{<\omega} \subseteq Y$. If $X$ is a set of ordinals then $\bar{X}$ denotes the topological closure of $X$ in the order topology. 


\section{The main result}

The purpose of this section is to show that MRP implies that $\lambda^{\omega}=\lambda$, for every cardinal $\lambda>\omega_{1}$ with $\operatorname{cof}(\lambda)>\omega$. We start by recalling the relevant definitions from [10].

Definition 1 Let $\theta$ be a regular cardinal, let $X$ be uncountable, and let $M \prec H(\theta)$ be countable such that $[X]^{\omega} \in M$. A subset $\Sigma$ of $[X]^{\omega}$ is $M$-stationary if for all $E \in M$ such that $E \subseteq[X]^{\omega}$ is club, $\Sigma \cap E \cap M \neq \emptyset$.

Recall that the Ellentuck topology on $[X]^{\omega}$ is obtained by declaring a set open if it is the union of sets of the form

$$
[x, N]=\left\{Y \in[X]^{\omega}: x \subseteq Y \subseteq N\right\}
$$

where $N \in[X]^{\omega}$ and $x \subseteq N$ is finite.

Definition $2 \Sigma$ is an open stationary set mapping if there is an uncountable set $X$ and a regular cardinal $\theta$ such that $[X]^{\omega} \in H(\theta)$, the domain of $\Sigma$ is a club in $[H(\theta)]^{\omega}$ of countable elementary submodels $M$ such that $X \in M$ and for all $M, \Sigma(M) \subseteq[X]^{\omega}$ is open in the Ellentuck topology on $[X]^{\omega}$ and $M$-stationary.

The Mapping Reflection Principle (MRP) asserts that:

If $\Sigma$ is an open stationary set mapping, there is a continuous $\in$-chain $\vec{N}=\left(N_{\xi}: \xi<\omega_{1}\right)$ of elements in the domain of $\Sigma$ such that for all limit ordinals $\xi<\omega_{1}$ there is $\nu<\xi$ such that $N_{\eta} \cap X \in \Sigma\left(N_{\xi}\right)$ for all $\eta$ such that $\nu<\eta<\xi$.

If $\left(N_{\xi}: \xi<\omega_{1}\right)$ satisfies the conclusion of MRP for $\Sigma$ then it is said to be a reflecting sequence for $\Sigma$. It is shown in [10] that MRP is a consequence of PFA. We are now ready to prove the following theorem.

Theorem 1 Assume MRP. Then $\lambda^{\aleph_{0}}=\lambda$, for every $\lambda \geq \omega_{2}$ of uncountable cofinality.

Proof: We will prove the theorem by induction. The base case $\lambda=\aleph_{2}$ is handled by Moore's result [10] that MRP implies $2^{\aleph_{0}}=\aleph_{2}$. If $\lambda=\kappa^{+}$with $\operatorname{cof}(\kappa)>\omega$ then $\lambda^{\aleph_{0}}=\lambda \cdot \kappa^{\aleph_{0}}$, so the result holds by the inductive hypothesis. If $\lambda$ is a limit cardinal and $\operatorname{cof}(\lambda)>\omega$ then 
$\lambda^{\aleph_{0}}=\sup \left\{\mu^{\aleph_{0}}: \mu<\lambda\right\}$, so the result also follows by the inductive hypothesis. Thus, the only interesting case is when $\lambda=\kappa^{+}$, with $\kappa$ singular of countable cofinality. In this case we will show, using MRP, that $\kappa^{\aleph_{0}}=\kappa^{+}$.

Now, let $\kappa$ be singular of countable cofinality and assume the theorem holds below $\kappa$. Fix a sequence $\left(C_{\delta}: \delta \in \kappa^{+}\right)$such that $C_{\delta}$ is a club in $\delta$ of minimal order type. In fact, we will be interested only in ordinals $\delta$ of cofinality $\leq \omega_{1}$. For every pair of ordinals $\delta, \beta<\kappa^{+}$, we fix a decomposition $\delta=\bigcup_{n} K(n, \delta, \beta)$ such that:

(i) $|K(n, \delta, \beta)|<\kappa$, for all $n$

(ii) $K(n, \delta, \beta) \subseteq K(m, \delta, \beta)$, for $n<m$

(iii) if $\eta<\beta$ is of cofinality $\omega_{1}$ then there is $n$ such that $C_{\eta} \cap \delta \subseteq$ $K(n, \delta, \beta)$

(iv) $K(n, \delta, \beta)$ is a closed subset of $\delta$, for all $n$.

This is easily achieved, for example, as follows. First of all, fix an increasing sequence $\left(\kappa_{n}: n \in \omega\right)$ of regular cardinals converging to $\kappa$. For all $\eta<\kappa^{+}$let $\phi_{\eta}: \kappa \rightarrow \eta$ be a surjection. Now set:

$$
K(n, \delta, \beta)=\delta \cap \overline{\phi_{\delta}\left[\kappa_{n}\right] \cup \bigcup\left\{C_{\eta} \cap \delta: \eta \in \phi_{\beta}\left[\kappa_{n}\right] \& \operatorname{cof} \eta=\omega_{1}\right\}} .
$$

Fix also a partition $\left\{A_{s}: s \in \kappa^{<\omega}\right\}$ of $\left\{\delta<\kappa^{+}: \operatorname{cof}(\delta)=\omega\right\}$ into disjoint stationary sets. Let $D(n, \delta, \beta)$ be the set of all $g \in \kappa^{\omega}$ such that there are infinitely many $j$ such that $K(n, \delta, \beta) \cap A_{g \nmid j} \neq \emptyset$. Using the fact that $K(n, \delta, \beta)$ is of size $<\kappa$ and the inductive hypothesis we immediately have the following.

Fact $1 D(n, \delta, \beta)$ is of size smaller than $\kappa$, for all $n, \delta$ and $\beta$.

We will be done once we show the following.

Lemma 1 Assume MRP. Then $\bigcup\{D(n, \delta, \beta): n<\omega$ and $\delta, \beta<$ $\left.\kappa^{+}\right\}=\kappa^{\omega}$.

Proof: Fix $g \in \kappa^{\omega}$. We have to find some $(n, \delta, \beta)$ such that $g \in$ $D(n, \delta, \beta)$. We are going to define an open stationary set mapping $\Sigma_{g}$ and apply MRP. We first fix some notation. Given a countable set $X$, we let $\delta_{X}=\sup \left(X \cap \kappa^{+}\right)$and $\alpha_{X}=\sup \left(X \cap \omega_{1}\right)$. If $\alpha<\gamma<\omega_{1}$, let the 
height of $\alpha$ in $\gamma$ be defined by $h t_{\gamma}(\alpha)=\left|C_{\gamma} \cap \alpha\right|$. Fix a sufficiently large regular cardinal $\theta$. Suppose $M$ is a countable elementary submodel of $H(\theta)$ containing all the relevant information. Fix $\beta_{M}<\kappa^{+}$large enough such that for every $\gamma<\kappa^{+}$of cofinality $\omega_{1}$ there is $\eta<\beta_{M}$ of cofinality $\omega_{1}$ such that $C_{\gamma} \cap M=C_{\eta} \cap M$. If $\gamma \in M \cap \kappa^{+}$let $n_{\gamma}$ be the smallest integer $l$ such that $\gamma \in K\left(l, \delta_{M}, \beta_{M}\right)$. Now, let $\Sigma_{g}(M)$ be the set of all $X \in\left[M \cap \kappa^{+}\right]^{\omega}$ such that $\alpha_{X}<\alpha_{M}, \delta_{X}<\delta_{M}$ and such that letting $m=h t_{\alpha_{M}}\left(\alpha_{X}\right)$, we have that $A_{g \mid m} \cap K\left(n_{\delta_{X}}, \delta_{M}, \beta_{M}\right) \neq \emptyset$. We will show that $\Sigma_{g}(M)$ is open and $M$-stationary, for all $M$.

Claim $1 \Sigma_{g}(M)$ is open.

Proof: Suppose $X \in \Sigma_{g}(M)$. First find $\alpha \in X \cap \omega_{1}$ such that $h t_{\alpha_{M}}(\alpha)=h t_{\alpha_{M}}\left(\alpha_{X}\right)=m$. Let $n=n_{\delta_{X}}$. By the definition of $n_{\delta_{X}}$ we have that $\delta_{X} \notin K\left(n-1, \delta_{M}, \beta_{M}\right)$. Since $K\left(n-1, \delta_{M}, \beta_{M}\right)$ is a closed subset of $\delta_{M}$, there is a $\gamma$ below $\delta_{X}$ such that $\left(\gamma, \delta_{X}\right] \cap$ $K\left(n-1, \delta_{M}, \beta_{M}\right)=\emptyset$. Pick $\delta \in X$ larger than $\gamma$. Then we have the following.

Subclaim $1[\{\alpha, \delta\}, X] \subseteq \Sigma_{g}(M)$

Proof: If $Y \in[\{\alpha, \delta\}, X]$, clearly, we have that $h t_{\alpha_{M}}\left(\alpha_{Y}\right)=m$. Since $\delta \in Y$ and $Y \subseteq X$ we have that $\delta \leq \delta_{Y} \leq \delta_{X}$. By the above remarks we can conclude that $n=n_{\delta_{X}} \leq n_{\delta_{Y}}$. Now, since $X \in \Sigma_{g}(M)$, $K\left(n_{\delta_{X}}, \delta_{M}, \beta_{M}\right) \cap A_{g\lceil m} \neq \emptyset$. By construction, $K\left(n_{\delta_{X}}, \delta_{M}, \beta_{M}\right) \subseteq$ $K\left(n_{\delta_{Y}}, \delta_{M}, \beta_{M}\right)$ so $K\left(n_{\delta_{Y}}, \delta_{M}, \beta_{M}\right) \cap A_{g \nmid m} \neq \emptyset$. Since $h t_{\alpha_{M}}\left(\alpha_{Y}\right)=m$, $Y \in \Sigma_{g}(M)$.

Claim $2 \Sigma_{g}(M)$ is M-stationary.

Proof: Given $f:\left[\kappa^{+}\right]^{<\omega} \rightarrow \kappa^{+}$belonging to $M$, we must find $X \in$ $M \cap \Sigma_{g}(M)$ which is closed under $f$. First, find $N \in M$, a countable elementary submodel of $H\left(\kappa^{++}\right)$containing all the relevant objects for the argument below. Let $h t_{\alpha_{M}}\left(\alpha_{N}\right)=m$ and find $\alpha \in N$ with the same height in $\alpha_{M}$. Now let $C$ be the set of $\delta<\kappa^{+}$such that $f[\delta]^{<\omega} \subseteq \delta$. Then $C$ is a club subset of $\kappa^{+}$and $C \in N$. Since $A_{g \nmid m}$ is stationary in $\kappa^{+}$and, by our assumption it belongs to $N$, we can find $\delta \in C \cap A_{g \nmid m} \cap N$. Then $\delta \in K\left(n_{\delta}, \delta_{M}, \beta_{M}\right) \cap A_{g \nmid m}$. Finally, let 
$Z \in N$ be a countable set cofinal in $\delta$ and let $X$ be the $f$-closure of $\{\alpha\} \cup Z$. Then $\delta_{X}=\delta$ and

$$
m=h t_{\alpha_{M}}(\alpha) \leq h t_{\alpha_{M}}\left(\alpha_{X}\right) \leq h t_{\alpha_{M}}\left(\alpha_{N}\right)=m .
$$

Since $K\left(n_{\delta_{X}}, \delta_{M}, \beta_{M}\right) \cap A_{g \mid m}$ is nonempty, $X \in \Sigma_{g}(M) \cap M$ and $X$ is closed under $f$.

Let $\left(M_{\eta}: \eta<\omega_{1}\right)$ be a reflecting sequence for $\Sigma_{g}$ provided by MRP. Let $N=\bigcup_{\eta} M_{\eta}$ and $\delta=\sup \left(N \cap \kappa^{+}\right)$. Let $\delta_{\eta}=\sup \left(M_{\eta} \cap \kappa^{+}\right)$, for every $\eta<\omega_{1}$. We find a club $E \subseteq \omega_{1}$ such that $\left\{\delta_{\eta}: \eta \in E\right\} \subseteq C_{\delta}$ and $M_{\eta} \cap \omega_{1}=\eta$, for all $\eta \in E$. Let $\alpha$ be a limit point of $E$. For the rest of this proof let $M=M_{\alpha}$. Now $C_{\delta} \cap M=C_{\gamma} \cap M$ for some $\gamma<\beta_{M}$, by the choice of $\beta_{M}$. By condition (iii) on $K\left(i, \delta_{M}, \beta_{M}\right)$ there is an $n$ such that $C_{\gamma} \cap \delta_{M}$ is a subset of $K\left(n, \delta_{M}, \beta_{M}\right)$. Since $C_{\gamma} \cap M \subseteq C_{\gamma} \cap \delta_{M}$, we can conclude that $C_{\delta} \cap M$ is a subset of $K\left(n, \delta_{M}, \beta_{M}\right)$.

Let $\nu<\alpha$ be such that $M_{\eta} \in \Sigma_{g}(M)$, for all $\eta$ such that $\nu<\eta<\alpha$. For any such $\eta \in E, M_{\eta} \in M$, so $\delta_{\eta} \in C_{\delta} \cap M \subseteq K\left(n, \delta_{M}, \beta_{M}\right)$. If $\eta \in E$ and $h t_{\alpha_{M}}(\eta)=j$, then $A_{g \nmid j} \cap K\left(n_{\delta_{\eta}}, \delta_{M}, \beta_{M}\right) \neq \emptyset$ and, since $n_{\delta_{\eta}} \leq n$, we have that $K\left(n_{\delta_{\eta}}, \delta_{M}, \beta_{M}\right) \subseteq K\left(n, \delta_{M}, \beta_{M}\right)$. Now, for any $i$ we can find an $\eta \in E$ such that $\nu<\eta<\alpha$ and $h t_{\alpha_{M}}(\eta) \geq i$, so there are infinitely many $j$ such that $A_{g \nmid j} \cap K\left(n, \delta_{M}, \beta_{M}\right) \neq \emptyset$, i.e. $g \in D\left(n, \delta_{M}, \beta_{M}\right)$, as desired.

Now we have the following immediate corollary.

Corollary 1 PFA implies SCH.

Proof: This follows by induction. By Silver's theorem [12] the first cardinal violating $\mathrm{SCH}$ cannot be singular strong limit of uncountable cofinality. On the other hand, if $\kappa$ is a singular strong limit cardinal of countable cofinality then, by Theorem $1,2^{\kappa}=\kappa^{\aleph_{0}}=\kappa^{+}$.

\section{Final remarks and side results}

The techniques presented in the previous sections can be applied to investigate another interesting problem in the area of forcing axioms. Since forcing axioms have been able to settle many of the classical 
problems of set theory, we can expect that the models of a forcing axiom are in some sense canonical. There are many ways in which one can give a precise formulation to this concept. For example, one can study what kind of forcings can preserve PFA, or else if a model $V$ of a forcing axiom can have an interesting inner model $M$ of the same forcing axiom. There are many results in this area, some of them very recent. For instance, König and Yoshinobu [8, Theorem 6.1] showed that PFA is preserved by $\omega_{2}$-closed forcing. The same holds for BPFA. In fact, BPFA is preserved by any proper forcing that does not add subsets of $\omega_{1}$. In the other direction, in [14] Veličković showed that if MM holds and $M$ is an inner model such that $\omega_{2}^{M}=\omega_{2}$, then $\mathcal{P}\left(\omega_{1}\right) \subseteq M$ and in a very recent paper Caicedo and Veličković [1] showed that if $M \subseteq V$ are models of BPFA and $\omega_{2}^{M}=\omega_{2}$ then $\mathcal{P}\left(\omega_{1}\right) \subseteq M$. Their argument also shows that if $M \subseteq V$ are models of MRP and $\omega_{2}^{M}=\omega_{2}$, then $\mathcal{P}(\omega) \subseteq M$. We can use the result of the previous section combined with this last result to show that PFA is destroyed by many of the cardinal preserving notions of forcing which add new $\omega$-sequences. A result of this sort has been obtained by Moore in [11].

Theorem 2 Let $V$ and $W$ be two models of set theory with the same cardinals with $V \subseteq W$. Assume $V$ and $W$ are both models of MRP and that, moreover, for every cardinal $\kappa$, stationary subsets in $V$ of $\left\{\alpha \in \kappa^{+}: \operatorname{cof} \alpha=\omega\right\}$ are also stationary in $W$. Then $V$ and $W$ have the same $\omega$-sequences of ordinals.

Proof: Assume otherwise. We proceed by induction on the least cardinal $\kappa$ such that there in an $\omega$-sequence of elements of $\kappa$ which is in $W$, but not in $V$. The base case $\omega$ is handled by the above result of Caicedo and Veličković. We now run into two cases: either the least such $\kappa$ has countable cofinality in $V$ or it doesn't. The more involved case appears when $\operatorname{cof}^{V} \kappa>\omega$. We present in some detail how to prove the induction step in this situation. With minor modifications the reader can supply the proof for the case that $\kappa$ is of countable cofinality. The idea is to redo the proof of the previous section using a $g \in \kappa^{\omega} \backslash V$. However some extra care has to be paid in the definition of the sets $K(n, \delta, \beta)$. Let $\left\{A_{s}: s \in \kappa^{<\omega}\right\} \in V$ be a partition of the points of countable $V$-cofinality of $\kappa^{+}$into disjoint stationary sets. By the assumptions each $A_{s}$ is still stationary in $W$. Fix $\left(E_{\delta}: \delta<\kappa^{+} \& \operatorname{cof}^{V} \delta \geq \omega_{1}\right) \in V$ such that for all $\delta$ in its domain, 
$E_{\delta}$ is a club in $\delta$ of minimal $V$-order-type. So for each $\delta, E_{\delta}$ has order type at most $\kappa$. Define in $V$, for all $\alpha<\kappa$ and $\delta, \beta<\kappa^{+}$, sets $K(\alpha, \delta, \beta)$ such that $\delta=\bigcup_{\alpha} K(\alpha, \delta, \beta)$ and:

(i) $|K(\alpha, \delta, \beta)|<\kappa$

(ii) $K(\alpha, \delta, \beta) \subseteq K(\gamma, \delta, \beta)$ for $\alpha<\gamma$

(iii) if $\eta<\beta$ and $\operatorname{cof}^{V} \eta \geq \omega_{1}$ then there is $\alpha$ such that $E_{\eta} \cap \delta \subseteq$ $K(\alpha, \delta, \beta)$

(iv) $K(\alpha, \delta, \beta)$ is a closed subset of $\delta$.

This is easily achieved, for example, as follows. For all $\eta \in\left[\kappa, \kappa^{+}\right)$let $\phi_{\eta}: \kappa \rightarrow \eta$ be a bijection. Now set:

$$
K(\alpha, \delta, \beta)=\delta \cap \overline{\phi_{\delta}[\alpha] \cup \bigcup\left\{E_{\eta} \cap \delta: \eta \in \phi_{\beta}[\alpha] \&\left|E_{\eta} \cap \delta\right| \leq|\alpha|\right\}} .
$$

Define $D(\alpha, \delta, \beta)$ to be the set of all $g \in \kappa^{\omega}$ such that there are infinitely many $j$ such that $A_{g \nmid j} \cap K(\alpha, \delta, \beta) \neq \emptyset$ and use the inductive hypothesis to get that $D(\alpha, \delta, \beta)^{V}=D(\alpha, \delta, \beta)^{W}$. Since $\kappa$ is the least cardinal with a new $\omega$-sequence, it follows that $\operatorname{cof}^{W}(\kappa)=\omega$. Let $g=\left(\alpha_{n}: n \in \omega\right) \in W$ be cofinal in $\kappa$. From now on work in $W$. Let $K(n, \delta, \beta):=K\left(\alpha_{n}, \delta, \beta\right)$. Now as in the previous section use the parameters

$$
\left\{K(n, \delta, \beta): n<\omega \& \delta, \beta<\kappa^{+}\right\},\left\{A_{s}: s \in \kappa^{<\omega}\right\}, g
$$

to define $\Sigma_{g}$ and show that it is an open stationary set mapping. We also refer to the previous section for the notation.

Now, apply MRP to $\Sigma_{g}$ and using exactly the same argument as in the previous section show that $g \in D\left(\alpha_{n},, \delta_{M}, \beta_{M}\right)$ for some $M$ in a reflecting sequence for $\Sigma_{g}$. Some extra care has to be paid since if $\left(M_{\eta}: \eta<\omega_{1}\right)$ is a reflecting sequence provided by MRP and $\delta=$ $\sup _{\eta<\omega_{1}} \delta_{M_{\eta}}, \delta$ may have $V$-cofinality larger than $\omega_{1}$. However we have overcome the problem since we have defined the sets $K(\alpha, \delta, \beta)$ more carefully than in the previous section.

In fact the theorem can be proved under the milder assumptions that $V$ and $W$ have the same cardinals, the same reals, $W \models$ MRP and, for every cardinal $\kappa$, there is in $V$ a partition $\left\{A_{s}: s \in \kappa^{<\omega}\right\}$ of the points of $\kappa^{+}$of countable $V$-cofinality into disjoint sets which are stationary in $W$. By a recent result of Larson building on ideas of 
Todorčević it is known that such partitions can be found in ZFC for $\kappa=\omega$ just assuming that $\omega_{1}^{V}=\omega_{1}^{W}$. It is open whether for higher cardinals such partitions exists in ZFC. A positive answer to this question would entail that if $V \subseteq W$ are models with the same reals and cardinals and $W \models$ MRP then $O R D^{\omega} \cap V=O R D^{\omega} \cap W$.

We also remark that what we need to run the proof of theorem 1 is the following weak form of reflection for stationary sets:

Let $\kappa$ be of countable cofinality. Then for every countable family $\left\{A_{n}: n \in \omega\right\}$ of stationary sets of points of countable cofinality of $\kappa^{+}$there are $n, \delta, \beta$ and there are infinitely many $j$ such that $A_{j} \cap K(n, \delta, \beta)$ is not empty.

It is possible to see that this "Reflection Principle" holds above a strongly compact cardinal. What we have shown in this paper is that this property holds also under MRP.

Acknowledgements: The results presented in this paper are a part of the Ph.D. thesis which I am completing under the direction of professors Alessandro Andretta of the University of Torino and Boban Veličković of the University Paris 7. Part of this research has been completed while visiting the Erwin Schrödinger International Institute for Mathematical Physics during the Fall of 2004 in the framework of the ESI Junior Research Fellowship 2004. I wish to thank Boban Veličković who suggested me to investigate the effects of PFA on the arithmetic of singular cardinals and who is guiding my steps in the study of forcing axioms. I thank also Andrés Caicedo and Justin Moore for many useful comments and suggestions.

\section{References}

[1] A. Caicedo, B. Veličković, Bounded proper forcing axiom and well orderings of the reals, preprint, 18 pages

[2] J. Cummings, E. Schimmerling, Indexed squares, Israel J. Math., 131, 2002, pp. 61-99

[3] W. B. Easton, Powers of regular cardinals, Ann. Math. Logic, vol. 1, 1970, pp. 139-178 
[4] M. Foreman, M. Magidor, S. Shelah, Martin's Maximum, saturated ideals and nonregular ultrafilters, Ann.of Math. (2), vol. 127(1), 1988, pp. 1-47

[5] M. Gitik, Prikry Type Forcings, Handbook of Set Theory, Foreman, Kanamori, Magidor (editors), to appear

[6] M. Gitik, Blowing up power of a singular cardinal - wider gaps, Ann. Pure and Applied Logic, vol. 116, 2002, pp. 1-38

[7] T. Jech, Set theory: the Millennium edition, Springer Verlag, 2003

[8] B. König, Y. Yoshinobu. Fragments of Martin's Maximum in generic extensions, Mathematical Logic Quarterly 50 (3) (2004), pp. 297-302

[9] M. Magidor, Lectures on weak square principles and forcing axioms, unpublished notes of the course held in the Jerusalem Logic Seminar, summer 1995

[10] J. T. Moore, Set mapping reflection, J. Math. Log. vol. 5(1), 2005, pp. $87-97$

[11] J. T. Moore, The Proper Forcing Axiom, Prikry forcing, and the Singular Cardinals Hypothesis, to appear in Annals of Pure and Applied Logic

[12] J. H. Silver, On the singular cardinal problem, Proceedings of the International Congress of Mathematicians, Vancouver, B.C., 1974, Vol.1, Canad. Math. Congress, Montreal, Que., 1975, pp. 265-268

[13] R.M. Solovay, Strongly compact cardinals and the GCH, Proccedings of the Tarski Symposium, L.Henkin et al. editors, Proc. Sympos. Pure Math., Vol XXV, Univ. of California, Berkeley, Calif., 1971. Amer. Math. Soc., Providence, R.I., 1974, pp. 365-372

[14] B. Veličković, Forcing axioms and stationary sets, Adv.Math., vol. 94(2), 1992, pp. 256-284 